\title{
Serum carboxymethyl-lysine, a dominant advanced glycation end product, is increased in women with gestational diabetes mellitus
}

\author{
Vendula Bartakova ${ }^{a}$, Radana Kollarova ${ }^{b}$, Katarina Kuricovaa ${ }^{a}$ Katarina Sebekova ${ }^{b}$, Jana Belobradkova ${ }^{c}, K^{2}$ aterina Kankova ${ }^{a}$
}

\begin{abstract}
Aims. The objective of the study was to measure one of the circulating Advanced Glycation End Products (AGEs) - NE(carboxymethyl)lysine $(\mathrm{CML})$ - in a case-control study $(\mathrm{n}=307)$ of pregnant women with gestational diabetes mellitus (GDM) and physiological pregnancies and to ascertain the factors contributing to CML levels and the potential relevance of CML for selected perinatal and postpartum outcomes.

Methods. All subjects underwent oGTT between $24^{\text {th }}$ and $30^{\text {th }}$ week of gestation and GDM was diagnosed according to WHO criteria. CML was determined by ELISA using commercial kit.

Results. Unadjusted and plasma protein adjusted CML levels were significantly higher in women with GDM compared to healthy controls ( $P=0.00043$ and $P=1 \times 10^{-5}$, respectively, Mann-Whitney). CML was significantly inversely correlated with both pre- and mid-gestational BMl, however, differences between GDM and control group remained significant even after adjustment for BMI. CML levels correlated with 1-h and 2-h post-load glycaemia during oGTT.

Conclusion. In conclusion, we found statistically significantly higher protein- and BMI-normalised CML levels measured during $24-30^{\text {th }}$ week of gestation in women with GDM compared to healthy pregnant controls. Further studies are warranted to comprehensively asses the spectrum of AGEs in GDM and their relevance to future metabolic health of mother and offspring.
\end{abstract}

Key words: gestational diabetes, CML, BMI, oral glucose tolerance test, postpartum glucose intolerance

Received: April 23, 2015; Accepted: September 10, 2015; Available online: November 24, 2015

http://dx.doi.org/10.5507/bp.2015.045

${ }^{a}$ Department of Pathophysiology, Faculty of Medicine, Masaryk University, Kamenice 5/A18, 62500 Brno, Czech Republic bInstitute of Molecular BioMedicine, Medical Faculty, Comenius University, Sasinkova 4, 81108 Bratislava, Slovak Republic 'Diabetes Centre of the Department of Internal Medicine - Gastroenterology, University Hospital Brno, Czech Republic Corresponding author:Vendula Bartakova, e-mail:vbartak@med.muni.cz

\section{INTRODUCTION}

Gestational diabetes mellitus (GDM) is a common complication of pregnancy whose incidence is rising worldwide for multiple reasons - a problem of overweight and obesity pertains to more and more younger people, babies are born to older mothers, and also screening programs contribute to increasing cumulative prevalence, no matter what criteria are being used for diagnostics ${ }^{1}$.

Normal pregnancy is characterized by a marked reduction in maternal insulin sensitivity in the second and third trimester. The decrease by $50 \%$ is comparable with that observed in type 2 diabetes mellitus (T2DM) and it is believed to be beneficial for foetal growth through facilitation of nutrient transport to the foetus. The ability of $\beta$-cells to compensate physiological increase of insulin resistance by matched increase of insulin secretion determines glucose tolerance. Reduced $\beta$-cell reserve or adaptation of the islets of Langerhans to higher insulin demand may lead to GDM development. The resulting abnormal metabolic situation during GDM pregnancy adversely influences the outcome (mainly due to macrosomia with subsequent delivery complications) and likely also the postnatal health status of offspring due to the foetal programming (increasing the risk of metabolic diseases such as obesity and T2DM later in life). Apart from established metabolic abnormalities assessed by routine laboratory assays, there are a host of recently identified disturbances (such as altered spectrum of adipokines, cytokines etc.) potentially contributing to the adverse consequences for both mother and baby ${ }^{2}$.

Advanced Glycation End products (AGEs), heterogeneous, long-lived protein adducts, produced by a nonenzymatic chemical reactions between sugars and their derivatives (such as methylglyoxal) and the amino groups of proteins ${ }^{3}$ are traditionally considered as a result of long-term dysregulation of glucose homeostasis. However detailed kinetic studies indicate a prompt reflexion of glucose metabolism ${ }^{4}$. From this point of view, thanks to the possibility of precisely determining the onset and subsequent cessation of the disease - GDM represents a highly relevant phenotype for studying acute changes of AGEs dynamics and its relationship to glucose metabolism.

$\mathrm{N} \varepsilon$-(carboxymethyl)lysine (CML) represents one of the dominant immunologically detectable forms of AGEs both in plasma/serum and in tissues. CML is routinely used as a proxy of a total load of AGEs in various pathological situations and increased CML levels have been repeatedly reported in patients with T2DM and related complications $^{5-7}$. To date, there are only very limited clinical data about AGE levels in GDM. Several studies of limited and heterogeneous sample size (often mixing pre- 
existing diabetic pregnant women and GDM) showed increased AGEs plasma levels in GDM compared to healthy pregnant women ${ }^{8-10}$. Two studies focused on tissue AGEs assessed by skin autofluorescence ${ }^{11,12}$ and found no difference between normal and GDM pregnancies. Only one study so far investigated foetal outcome in connection to AGE levels in humans ${ }^{9}$ and found higher maternal serum AGE levels in GDM women with abnormal foetal outcome compared to controls with normal foetal outcome. Finally, none of these studies specifically focused on CML in the GDM context.

We hypothesised that CML levels may rapidly increase in parallel to glycaemia at the time of GDM onset (or even before, in predisposed women with latent glucose metabolism abnormality) that CML levels in pregnancy might predict increased risk of adverse foetal outcome and persistence or early re occurrence of glucose abnormality postpartum. Therefore, the aims of the present study were: (i) to determine plasma mid-gestational CML levels in pregnant women with and without GDM, (ii) to investigate the correlation between CML levels, glycaemic indices, anthropometric parameters and other clinical parameters to determine the major contributors to CML level, (iii) to evaluate the relationship between CML levels and perinatal parameters and finally (iv) to ascertain the predictive potential of mid-gestational CML levels for postpartum glucose intolerance (i.e. prediabetes or manifest diabetes).

\section{MATERIALS AND METHODS}

\section{Subjects}

A total of 307 pregnant women who gave birth at the Gynaecology and Obstetrics clinics of the University Hospital Brno were included in the study. Of these $n$ $=222$ had GDM (those were consenting consecutive women followed from the time of GDM diagnosis till the birth at the Diabetes Centre of the same hospital) and $\mathrm{n}=85$ had physiological pregnancies (those were consenting non-diabetic women recruited from several outpatients prenatal centres in the city of Brno, who passed mid-gestational GDM screening with negative results). All subjects were Caucasians, Czech nationality and were geographically from the same area. Exclusion criteria were T1DM or T2DM before pregnancy, multiple pregnancy, non-Caucasian population, foreign nationality and severe comorbidities.

GDM screening was carried out using oGTT with 75 $\mathrm{g}$ of glucose performed between 24th and 30th week of pregnancy. The sample was recruited during 2012 and GDM diagnosis was established according to the WHO criteria recommended by the Czech Diabetes Society at that time: FPG $>5.6 \mathrm{mmol} / \mathrm{L}, 1-\mathrm{h}$ post-load glucose $>8.9$ $\mathrm{mmol} / \mathrm{L}$ and 2 -h post-load glucose $>7.8 \mathrm{mmol} / \mathrm{L}$ (any one of the above three cut-off values qualified for the GDM diagnosis).

In April 2014, the Czech Diabetes Society adopted new diagnostic criteria for GDM in accordance with the International Association of the Diabetes and Pregnancy Study Groups (IADPSG) recommendations ${ }^{13}$ with the fol-

Table 1. Clinical, anthropometric and laboratory characteristics of the study groups.

\begin{tabular}{|c|c|c|c|}
\hline Parameter & $\operatorname{GDM}(\mathrm{n}=182)$ & Controls $(n=36)$ & $P$ \\
\hline Age (years) & $33[30-35]$ & $31[28-33]$ & 0.045 \\
\hline Parity & $2[1-2]$ & $2[1-2]$ & NS \\
\hline Pre-gestational BMI & $24.80[21.61-28.52]$ & $21.15[20.08-26.88]$ & 0.013 \\
\hline Weight gain in pregnancy $(\mathrm{kg})$ & $8[6-10]$ & $13[11-16]$ & $>1 \times 10^{-6}$ \\
\hline Offspring birth weight (g) & $3180[2910-3500]$ & $3360[3085-3650]$ & NS \\
\hline Macrosomia (>4000 g) (\%) & 6.6 & 8.3 & NS \\
\hline Family history of DM (\%) & 70.3 & 22.2 & 0.0019 \\
\hline Pregnancy after IVF (\%) & 7 & 0 & NS \\
\hline Insulin therapy in pregnancy (\%) & 28 & - & - \\
\hline Pre-gestational hypothyroidism (\%) & 11 & 5.5 & NS \\
\hline Pre-gestational hypertension (\%) & 5.5 & 5.5 & NS \\
\hline P.p. glucose abnormality (\%) & 6 & - & - \\
\hline \multicolumn{4}{|c|}{ Mid-gestation parameters (24-30 $0^{\text {th }}$ week of gestation) } \\
\hline oGTT FPG (mmol/L) & $4.7[4.4-5.0]$ & $4.1[4.0-4.4]$ & $>1 \times 10^{-6}$ \\
\hline oGTT 1-h post-75 g load (mmol/L) & $9.3[8.9-10.0]$ & $5.9[5.4-6.4]$ & $>1 \times 10^{-6}$ \\
\hline oGTT 2-h post-75 g load (mmol/L) & $8.1[7.7-8.8]$ & $5.5[4.8-6.0]$ & $>1 \times 10^{-6}$ \\
\hline $\mathrm{AUC}_{\mathrm{oGTT}}(\mathrm{mmol} / \mathrm{L} / \mathrm{h})$ & $13.0[12.3-13.6]$ & $9.5[8.8-10.1]$ & $>1 \times 10^{-6}$ \\
\hline BMI & $27.38[24.16-30.66]$ & $21.15[20.26-25.79]$ & 0.023 \\
\hline CML (ng/mL) & $683.64[560.00-805.30]$ & $507.54[433.56-679.50]$ & 0.00043 \\
\hline CML / plasma protein $(\mathrm{ug} / \mathrm{g})$ & $10.18[8.09-12.22]$ & $5.73[5.29-8.66]$ & 0.00001 \\
\hline CML/plasma protein/BMI & $0.38[0.27-0.47]$ & $0.26[0.18-0.38]$ & 0.0004 \\
\hline
\end{tabular}

Data expressed as a median [IQR] or proportions. Differences evaluated by nonparametric Mann-Whitney or chi-square test, respectively. Abbreviations: P.p. - postpartum. 
lowing thresholds: FPG $\geq 5.1 \mathrm{mmol} / \mathrm{L}, 1-\mathrm{h}$ post-load glucose: $\geq 10.0 \mathrm{mmol} / \mathrm{L}, 2$-h post-load glucose: $\geq 8.5 \mathrm{mmol} / \mathrm{L}$ retrospectively.

Recommended postpartum oGTT in GDM subjects ( 6 weeks - 12 months postpartum) was attended by $82 \%$ of women $(n=182)$ in Diabetes centre of the University Hospital Brno. Elective postpartum check-up was attended by $42.3 \%$ of non-GDM women $(n=36)$ who provided complete perinatal and postpartum anamnestic data but who did not undergo the postpartum oGTT. Hence, the complete set of mid-gestational, perinatal and postpartum data was available for the sub-set of 218 pregnant women and most analyses were done on this sub-set. The relationship between mid-gestational CML levels and parameters assessed at this time-point were also determined in the larger set of 307 pregnant women. Detailed characteristics of the subgroup with compete data are shown in Table 1. The study was approved by the Ethics Committee of Faculty of Medicine, Masaryk University and was conducted in accordance with the latest Helsinki declaration.

\section{Blood samples and CML measurement}

Samples of peripheral blood were taken from each participant between the 24th and 30th week of pregnancy (by a gynaecologist during their scheduled visit in the prenatal centre in non-GDM subjects or by a diabetologist during their first visit to the Diabetes Centre). Plasma was separated by centrifugation $\left(12850 \mathrm{~g}, 10 \mathrm{~min}, 4^{\circ} \mathrm{C}\right)$ and stored at $-70{ }^{\circ} \mathrm{C}$ until analysis. Total $\mathrm{CML}$ concentration in plasma was determined using an ELISA assay (MicroCoat Biotechnologie GmbH, Bernried, Germany), after pre-treatment of the samples with proteinase $\mathrm{K}$
(Roche, Mannheim, Germany) according to the manufacturer's instructions. Additionally plasma CML levels were expressed per $\mathrm{ng} / \mathrm{mL}$ of plasma proteins.

\section{Statistics}

Data are expressed as medians and interquartile ranges [IQR] or proportions for between-group comparisons. Nonparametric tests were used for comparison between groups (Mann-Whitney). Correlations were determined using Spearman correlation coefficients. The area under the glucose curve (AUC) was computed from oGTT values using the trapezoid rule ${ }^{14}$. Software Statistica was used for all analyses. $P<0.05$ was considered as statistically significant.

\section{RESULTS}

\section{CML levels in pregnant women with and without GDM}

Unadjusted plasma CML levels and plasma CML adjusted for total plasma protein (CML/protein) are shown in Fig. 1. In both cases, the values were significantly higher in women with GDM compared to healthy pregnant women (both $P<0.001$, Mann-Whitney $U$ test). As a confirmation, the same results were obtained when comparison was made in the larger sample of $n=307$ subjects with only mid-gestational data available (data not shown).

\section{Relationship between mid-gestational CML, glucose indices and BMI}

In the next step, we assessed correlations between unadjusted and adjusted plasma CML levels and all three
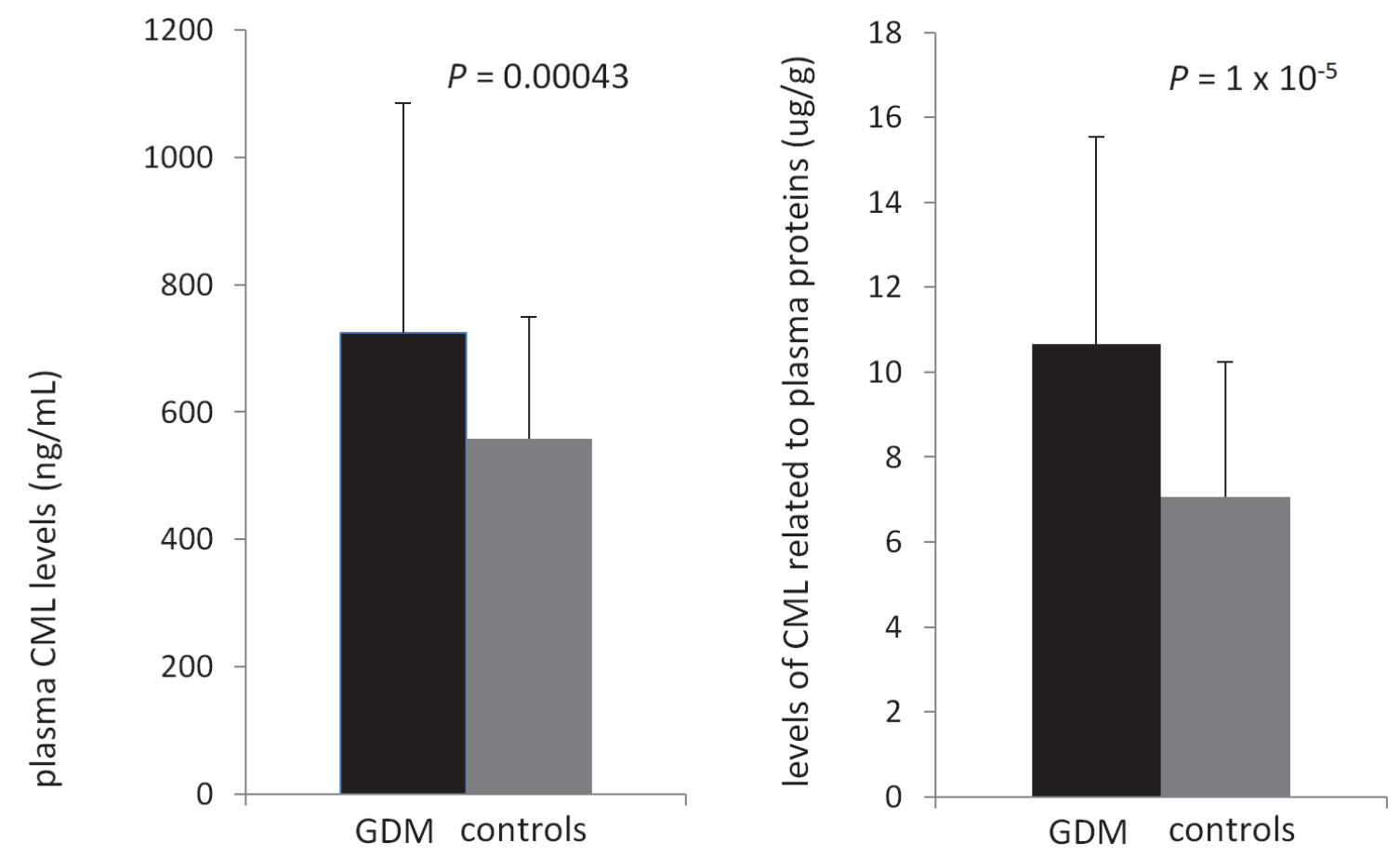

Fig. 1. Plasma unadjusted and total plasma protein adjusted CML in GDM women $(n=182)$ and healthy pregnant women $(n=36)$ in mid trimester of gravidity.

Data presented as mean \pm SD. Differences evaluated by Mann-Whitney. 
mid-gestational oGTT values $(P=$ NS for FPG, $\mathrm{r}=0.21$, $P=0.032$ and $\mathrm{r}=0.34, P=5.4 \times 10^{-4}$, respectively, for 1 -hr post-load glucose and $\mathrm{r}=0.16, P=0.016$ and $\mathrm{r}=0.22$, $P=0.001$, respectively for 2 -hr post-load glucose), pregestational BMI ( $\mathrm{r}=-0.27, P=5 \times 10^{-5}$ and $\mathrm{r}=-0.25, P=$ $1.3 \times 10^{-4}$, respectively) and mid-gestational BMI $(\mathrm{r}=-0.3$, $P=7 \times 10^{-6}$ and $\mathrm{r}=-0.27, P=4 \times 10^{-5}$, respectively). For all analyses Spearman correlation coefficient was used. Similar trends were found in the larger $(n=307)$ study group (data not shown).

Inverse correlation between CML and pre-and midgestational BMI confirmed our findings that CML levels are strongly affected by BMI in both children and adolescents ${ }^{15}$ and adults ${ }^{16}$. Other studies found the same relationship and specifically confirmed the presence of CML in adipose tissue $^{17,18}$. Taken together, it appears that CML is preferentially deposited in fat tissue and acts as an important mechanism involved in the dysregulation of adipokines in obesity, thereby contributing to the development of obesity-associated insulin resistance. In this study, both pre- and mid-gestational BMI inversely correlated with CML. For this reason and also given that both pre- and mid-gestational BMI differed between groups, we adjusted plasma CML/protein to BMI at the time of sampling, i.e. mid-gestational (see Table 1). Regardless of adjustments, CML levels remained significantly higher in the GDM group $(P=0.0004)$ and the significant difference was preserved in the larger group, $\mathrm{n}=307, P=0.04$ ).

\section{Relationship between CML levels and perinatal outcomes}

Plasma CML or CML/protein levels did not significantly correlate with offspring birth weight or pregnancy weight gain $(P=\mathrm{NS})$. Additionally, CML levels did not significantly differ between women whose babies had macrosomia and those without.

\section{Relationship between mid-gestational CML and postpartum glucose (in)tolerance}

Based on oGTT performed 6 weeks - 12 months after delivery using WHO criteria, the frequency of early postpartum glucose abnormality, i.e. impaired glucose tolerance (FPG 5.6 - $6.9 \mathrm{mmol} / \mathrm{L}$ or 2-h post-load glucose $7.8-11.0 \mathrm{mmol} / \mathrm{L})$ was $6 \%(\mathrm{n}=11)$ in our GDM sample. In spite of the limitations due to group size disparity, plasma $\mathrm{CML}, \mathrm{CML} /$ protein CML/protein/BMI in women with postpartum glucose intolerance did not significantly differ from those of postpartum glucose tolerant ex-GDM subjects $(P=\mathrm{NS})$. Further, glucose values during postpartum oGTT did not correlate with mid-gestational CML $(P=$ NS, Spearman).

\section{CML levels in pregnant women with and without GDM using IADPSG criteria}

Finally, to adjust for national changes in GDM diagnostic, we post hoc reclassified the study sample according to the new criteria. Using newly adopted IADPSG criteria, 125 women would be diagnosed as having GDM, 182 as healthy subjects (note all previously classified controls remained while 97 of previously diagnosed GDM subjects became controls) and then we compared CML levels. We found statistically significant differences in CML/BMI and $\mathrm{CML} /$ plasma protein/BMI adjusted levels $(\mathrm{P}=0.0014$ and $\mathrm{P}=0.014$ resp., Mann-Whitney) with levels significantly higher in the GDM group.

\section{DISCUSSION}

In this study, using a cohort of 307 metabolically wellcharacterized pregnant subjects, we found statistically significant differences in CML levels in the second trimester of pregnancy between pregnant women with and without GDM. At the same time, both unadjusted and plasma protein adjusted CML levels did not correlate with age, weight gain during pregnancy or offspring birth weight (both measured only in a subset of 218 women with complete postpartum data). However, they significantly inversely correlated with pre- and mid-gestational BMI and mid-gestational oGTT glucose in 1-h and 2-h post-75 g load. After adjustment of CML to mid-gestational BMI (due to the previous repeated findings of the inverse correlation between the two parameters and the significant difference in BMI between GDM and non-GDM group), the significant differences in CML between GDM and healthy pregnant women were preserved.

Several studies measured AGE levels in GDM patients during the second trimester of pregnancy. Buongioruo et al. ${ }^{10}$ found significantly higher AGE levels in women with diabetes in pregnancy compared to healthy controls. However, these authors included T1DM and T2DM pregnant women and the total number was 60 subjects. Guosheng et al. ${ }^{9}$ studied a comparable number of pregnant women as in our study and they ascertained differences in AGE levels between GDM and controls both at the beginning of second trimester and in late pregnancy but there was no significant difference between groups in terms of weight.

Interestingly, the results appear criteria-independent. Using IADPSG diagnostic criteria in the same study sample, differences in CML levels adjusted for BMI and total protein, remained statistically significant between women with GDM and controls. However, since the IADPSG criteria were applied post hoc, these results have to be considered hypothesis-driven and the conclusions speculative.

Our finding of significant correlation between 1- and 2-hr post-load glycaemia during oGTT is in line with the Baltimore longitudinal study of aging ${ }^{19}$ where increased CML levels were found in patients with abnormal 2-hr plasma glucose in oGTT compared to physiological 2-hr oGTT levels. No differences were found in CML levels for FPG.

The most important finding of our study is that although we confirmed previous findings of an inverse relationship between plasma CML levels and BMI (ref. ${ }^{15,16}$ ), women with GDM still had higher CML than non-GDM women. The implications of this metabolic alteration for foetal development and future metabolic and adipose pa- 
rameters are definitely worth future study in the light of the current data.

As in other studies, we found no statistically significant differences in CML levels in women with babies showing macrosomia compared to those with physiological newborns and no correlation was found between CML and birth weight. Guosheng et al. ${ }^{9}$ found higher AGE levels in GDM women with selected pathologies such as stillbirth, asphyxia or foetal malformation compared to those giving birth to normal infants. However these authors found no significant difference in AGE levels between women with large-for-gestational-age infants compared to those with normal weight either.

Finally, CML levels in the second trimester of pregnancy in our cohort were not predictive of early postpartum (6 weeks - 12 months after delivery) glucose abnormality. However this comparison may be invalid due to low numbers: only 11/182 GDM women were diagnosed with glucose abnormality in this time period. Nevertheless, as far as we are aware, this is a first study on CML levels in connection to persistence of glucose abnormality postpartum.

Limitations of this study include the fact that of multiple chemical entities representing AGEs, only circulating CML was measured. Since different metabolic pathways lead to the production of various classes of AGEs incl. CML, measuring a single AGE entity could be misleading. Fu et al. ${ }^{20}$ demonstrated that the major source of CML was created by lipid peroxidation, not glycation. Quantification of other non-CML AGEs is likely to be necessary for better understanding of the role of AGEs in GDM pathogenesis. Adipocytes express CD36, a scavenger receptor that binds AGEs (incl. CML) and facilitates endocytosis and degradation ${ }^{21,22}$. Thus, fat mass could influence circulating AGE concentrations through a role in the catabolism and breakdown of AGE. At the same time however, the CML/RAGE-axis appears to be a key modulator of obesity-induced dysregulation of adipokine expression in adipocytes and of subsequent insulin resistance ${ }^{17}$.

\section{CONCLUSION}

We found statistically significantly higher CML levels measured during the 24-30 $30^{\text {th }}$ week of gestation in women with GDM compared to healthy pregnant women. In spite of significant inverse correlation between CML and BMI, CML levels adjusted for BMI, were still significantly higher in GDM women. Mid-gestational CML levels were not predictive of perinatal complications such as macrosomia or early postpartum glucose abnormality in women with a GDM history. Further studies are warranted to more comprehensively asses the spectrum of AGEs in GDM and their relevance to the future metabolic health of mother and baby.

Acknowledgement: This study was supported by grant NT/11405 from Ministry of Health of Czech Republic.

Author contributions: BV: study design, data collection, statistical analyses and manuscript writing; KK, RK: bio- chemical analyses, data interpretation; JB: clinical data; KS: data interpretation; KK: final revision.

Conflict of interest statement: The authors state that there are no conflicts of interest regarding the publication of this article.

\section{REFERENCES}

1. Reece EA, Leguizamón G, Wiznitzer A. Gestational diabetes:The need for a common ground. Lancet 2009;373:1789-1797.

2. Lacroix M, Kina E, Hivert MF. Maternal/fetal determinants of insulin resistance in women during pregnancy and in offspring over life. Curr Diab Rep 2013;13:238-244.

3. Brownlee M, Cerami A, Vlassara H. Advanced glycosylation end products in tissue and the biochemical basis of diabetic complications. N Engl J Med 1988;318:1315-1321.

4. Uribarri J, Cai W, Ramdas M, Goodman S, Pyzik R, Chen X, Zhu L, Striker GE, Vlassara $H$. Restriction of advanced glycation end products improves insulin resistance in human type 2 diabetes: Potential role of ager1 and sirt1. Diabetes Care 2011;34:1610-1616.

5. Kalousová M, Skrha J, Zima T. Advanced glycation end-products and advanced oxidation protein products in patients with diabetes mellitus. Physiol Res 2002;51:597-604.

6. Boehm BO, Schilling S, Rosinger S, Lang GE, Lang GK, Kientsch-Engel $\mathrm{R}$, Stahl P. Elevated serum levels of n(epsilon)-carboxymethyl-lysine, an advanced glycation end product, are associated with proliferative diabetic retinopathy and macular oedema. Diabetologia 2004;47:1376-1379.

7. Schleicher ED, Wagner E, Nerlich AG. Increased accumulation of the glycoxidation product $\mathrm{n}$ (epsilon)-(carboxymethyl)lysine in human tissues in diabetes and aging. J Clin Invest 1997;99:457-468.

8. Harsem NK, Braekke K, Torjussen T, Hanssen K, Staff AC. Advanced glycation end products in pregnancies complicated with diabetes mellitus or preeclampsia. Hypertens Pregnancy 2008;27:374-386.

9. Guosheng L, Hongmei S, Chuan N, Haiying L, Xiaopeng Z, Xianqiong $L$. The relationship of serum age levels in diabetic mothers with adverse fetal outcome. J Perinatol 2009;29:483-488.

10. Buongiorno AM, Morelli S, Sagratella E, Castaldo P, Di Virgilio A, Maroccia E, Ricciardi G, Sciullo E, Cardellini G, Fallucca F, Sensi M. Levels of advanced glycosylation end-products (age) in sera of pregnant diabetic women: Comparison between type 1, type 2 and gestational diabetes mellitus. Ann Ist Super Sanita 1997;33:375-378.

11. de Ranitz-Greven WL, Bos DC, Poucki WK, Visser GH, Beulens JW, Biesma DH, de Valk HW. Advanced glycation end products, measured as skin autofluorescence, at diagnosis in gestational diabetes mellitus compared with normal pregnancy. Diabetes Technol Ther 2012;14:43-49.

12. de Ranitz-Greven WL, Kaasenbrood L, Poucki WK, Hamerling J, Bos DC, Visser GH, Biesma DH, Beulens JW, de Valk HW. Advanced glycation end products, measured as skin autofluorescence, during normal pregnancy and pregnancy complicated by diabetes mellitus. Diabetes Technol Ther 2012;14:1134-1139.

13. Sacks DA, Hadden DR, Maresh M, Deerochanawong C, Dyer AR, Metzger BE, Lowe LP, Coustan DR, Hod M, Oats JJ, Persson B, Trimble ER, Group HSCR. Frequency of gestational diabetes mellitus at collaborating centers based on iadpsg consensus panel-recommended criteria: The hyperglycemia and adverse pregnancy outcome (hapo) study. Diabetes Care 2012;35:526-528.

14. Matthews JN, Altman DG, Campbell MJ, Royston P. Analysis of serial measurements in medical research. BMJ 1990;300:230-235.

15. Sebeková K, Somoza V, Jarcusková M, Heidland A, Podracká L. Plasma advanced glycation end products are decreased in obese children compared with lean controls. Int J Pediatr Obes 2009;4:112-118.

16. Sebeková K, Krivošíková Z, Gajdoš M. Total plasma ne-(carboxymethyl)lysine and srage levels are inversely associated with a number of metabolic syndrome risk factors in non-diabetic youngto-middle-aged medication-free subjects. Clin Chem Lab Med 2014;52:139-149.

17. Semba RD, Arab L, Sun K, Nicklett EJ, Ferrucci L. Fat mass is inversely associated with serum carboxymethyl-lysine, an advanced glycation end product, in adults. J Nutr 2011;141:1726-1730. 
18. Gaens KH, Goossens GH, Niessen PM, van Greevenbroek MM, van der Kallen CJ, Niessen HW, Rensen SS, Buurman WA, Greve JW, Blaak EE, van Zandvoort MA, Bierhaus A, Stehouwer CD, Schalkwijk CG. $\mathrm{N} \varepsilon$-(carboxymethyl)lysine-receptor for advanced glycation end product axis is a key modulator of obesity-induced dysregulation of adipokine expression and insulin resistance. Arterioscler Thromb Vasc Biol 2014;34:1199-1208.

19. Semba RD, Beck J, Sun K, Egan JM, Carlson OD, Varadhan R, Ferrucci L. Relationship of a dominant advanced glycation end product serum carboxymethyl-lysine, and abnormal glucose metabolism in adults: The baltimore longitudinal study of aging. J Nutr Health Aging 2010;14:507-513.
20. Fu MX, Requena JR, Jenkins AJ, Lyons TJ, Baynes JW, Thorpe SROV. The advanced glycation end product, nepsilon-(carboxymethyl) lysine, is a product of both lipid peroxidation and glycoxidation reactions. J Biol Chem 1996;271:9982-9986.

21. Ohgami N, Nagai R, Ikemoto $M$, Arai $H$, Miyazaki A, Hakamata $H$, Horiuchi S, Nakayama H. Cd36, serves as a receptor for advanced glycation endproducts (age). J Diabetes Complications 2002;16:5659.

22. Kuniyasu A, Ohgami N, Hayashi S, Miyazaki A, Horiuchi S, Nakayama $\mathrm{H}$. Cd36-mediated endocytic uptake of advanced glycation end products (age) in mouse 3t3-I1 and human subcutaneous adipocytes. FEBS Lett 2003;537:85-90. 